\title{
Using Time Series Nighttime Images to Measure Light Pollution Trend in China
}

\author{
Wei Jiang, Guojin He, and Tengfei Long
}

\begin{abstract}
Rapid urbanization and economic development inevitably lead to light pollution, which has become a universal environmental issue. In order to reveal the change trend of light pollution in China, Images from 1992 to 2012 were selected from the Defense Meteorological Satellite Program Operational Linescan System (DMSP/OLS) and systematically corrected to ensure the consistency. Furthermore, we employed a linear regression trend method to measure China's light pollution trend across national scales. We found that: (1) China's light pollution expanded significantly in provincial capital cities over the past 21 years and hot-spots of light pollution were located in the eastern coastal region. The Yangtze River Delta, Pearl River Delta, and Beijing-Tianjin-Hebei regions have formed light pollution stretch areas.
\end{abstract}

Index Terms-Light pollution, nighttime light images, DMSP/OLS, time series, China.

\section{INTRODUCTION}

Light pollution was firstly raised by astronomers in 1930s [1]. Nowadays, it has become a widely-concerned environment issue following water pollution, air pollution and noise pollution [2]. Although night lighting facilities can benefit human activities at night, it has caused energy waste and directly or indirectly affect animals [3] and ecological environment [4], human health [5] and astronomical observation [1]. Since Chinese government implemented reform and opening up policy, unprecedented urbanization has taken place across the country with huge power consumption, and a large number of "sleepless city" have been formed. Due to lack of policy guidance and regulations, almost every large city has been threatened by light pollution. However, the issue of china's light pollution was not widely documented. Therefore, it is important to understand evolvement rules of china's light pollution to protect the ecological environment and improve the quality of life for urban residents.

Traditional field survey is costly and inefficient, difficult to be compared with historical data. However, earth observation technology of remote sensing has advantages of large scope, high frequency and long time series, especially nighttime light remote sensing technology can quickly access region and global nighttime light image [6]. Bennie et al.

Manuscript received June 10, 2017; revised August 12, 2017. This work was supported in part by the The National Key Research and Development Program of China -Rapid production method of large scale global change products (2016YFA0600302), Hainan Provincial Department of Science and Technology under Grant No.ZDKJ2016021 and ZDKJ2016015.

The authors are with the Institute of Remote sensing and Digital Earth, Chinese Academy of Sciences, Beijing 100094, China (e-mail jiangwei@radi.ac.cn, hegj@radi.ac.cn, and longtf@radi.ac.cn).
(2014) proposed to analyze contrast trends of light pollution across European continent and found that most countries in Europe were facing increasing lighting, while some countries like Novak, Ukraine and Belgium, the nighttime light is declining. Time-series nighttime light image was used to explore direct and indirect light pollution level in Athens suburban areas and the result demonstrated that light pollution increased significantly in the study area [7]. Meanwhile, nighttime light imageries were also applied to analyze light pollution in Pakistan.[8]. As to the research of the impact of light pollution on ecosystems, Kyba and Hoelker [9] confirmed the effect of night sky scattered light on biodiversity. In addition, Bennie et al. [7] evaluated global ecosystem types in exposure to artificial light combing DMSP/OLS images and land cover product and found that Mediterranean-climate ecosystem was affected greatest and global ecosystem have been localized and fragmented by artificially lights.

In order to understand china's light pollution trend during rapid economic and society development period, we investigated time-series DMSP/OLS nighttime light remote sensing imagery from 1992 to 2012 and employed linear regression method to measure the change trend of light pollution in China at national scale. This study conclusion can assist government to make decisions for light pollution environmental issues.

\section{Data PRocessing}

\section{A. Night-Time Light Imagery}

Long-term time-series DMSP/OLS nighttime light satellite images from 1992 to 2012 were selected as experimental data to study light pollution in China. The DMSP/OLS images in this study is version 4, which were downloaded from the National Geophysical Data Center (NGDC) website of National Oceanic and Atmospheric Administration (NOAA). The annual product of night-time lights contains frequency of cloud-free product, average light product and stable night-time light product respectively. We selected 33 periods of global stable night-time light product images as study dataset for it has removed background noises like gas flaring, wildlife fire and aurora. Therefore, the brightness in imagery represents the residential night-time lights. This imagery was given 30 arc second grids, spanning from -180 to 180 degrees longitude and from -65 to 75 degrees latitude and data pixel digital number(DN) ranges from 0-63 [10].

\section{B. Night-Time Lights Imagery Processing}

Because DMSO/OLS nighttime light imagery were acquired by six satellites (F10, F12, F14, F15, and F16) 
spanning 21 years without on-board calibration, it is necessary to correct nighttime light imagers to improve data comparability and accuracy [11]. In order to obtain research dataset for studying, we have made a workflow to process time-series nighttime light imageries (Fig. 1), and it mainly included three steps, namely, model establishment, image correction and post image processing.

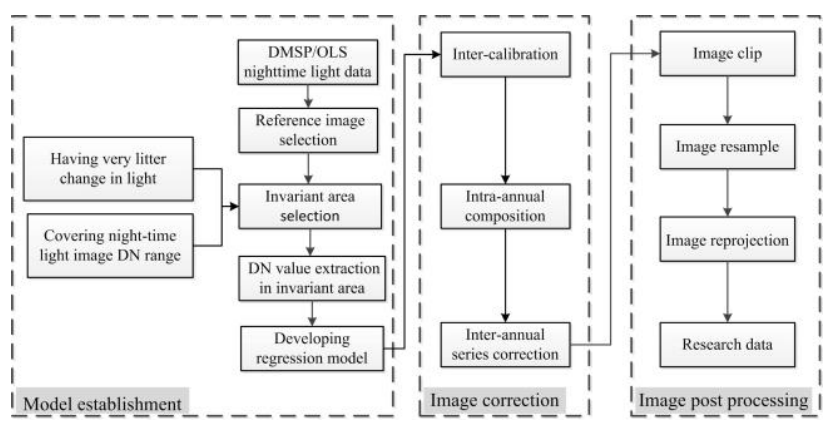

Fig. 1. The process flow of DMSP/OLS nighttime light imagery.

\section{METHOD}

Linear regression trend analysis was used to directly reveal temporal and spatial trends in each pixel with eliminating the DN variation error [12]. The calculation formula is as follows:

$$
R=\frac{\mathrm{t} \times \sum_{i=1}^{t} i \times D N_{i}-\sum_{i=1}^{t} i \sum_{i=1}^{t} D N_{i}}{\mathrm{t} \times \sum_{i=1}^{t} \dot{i}^{2}-\left(\sum_{i=1}^{t} i\right)^{2}}
$$

In the formula, $\mathrm{R}$ is a slope of the regression trend, $\mathrm{DN}_{\mathrm{i}}$ represents $\mathrm{DN}$ value in year i. $t$ represents time span. If $\mathrm{R}$ is above 0 , it shows that the $\mathrm{DN}$ value trend is increasing over the 21 years and the greater the $\mathrm{R}$ value, the more significant the increasing trend. However, if $\mathrm{R}$ is below 0 , it shows that the DN value is on the decline in 21 years and the smaller the $R$ value, the more significant the decreasing trend.

\section{RESULTS AND ANALYSIS}

\section{A. Comparison of Night Light Image Correction}

In order to evaluate the effect of nighttime light image correction, the TNL of china was extracted from different correction method to compare (Fig. 2). The TNL of different sensors among original images (Fig. 2 (a)) have significant difference in the same year. However, after the inter-calibration (Fig. 2 (b)), the TNL is more consistent in the cross year, it has showed that the sensor error was effectively eliminated. Furthermore, the inter-annual composition was employed to correct image (Fig. 2 (c)), the annual fluctuation of TNL showed increasing trend, but it has also sharply decreased in 2012. Thus, inter-annual series correction method was used to correct images and the correction result was showed in Fig. 2 (d). After inter-annual series correction, the increasing trend of TNL was more stable. It indicated that the image correction had good performance and it can be used to analyze the spatial and temporal trend of night light pollution in China.
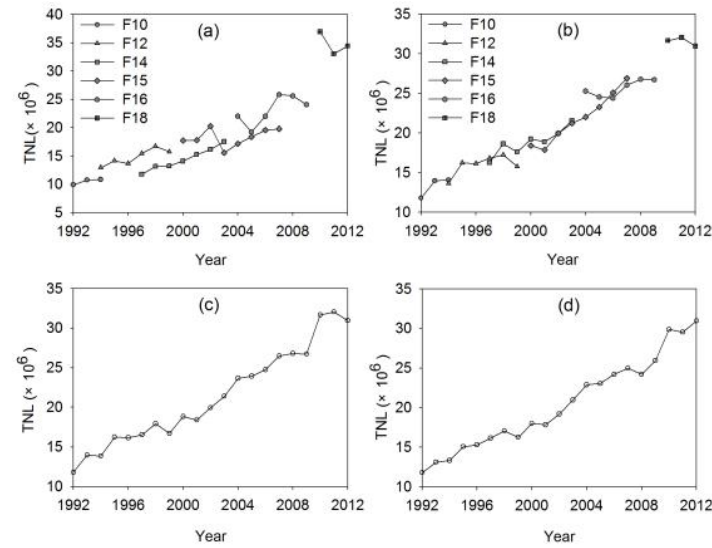

Fig. 2. TNL of nighttime light images before and after correction. (a) original images, (b) inter-calibration, (c) inter-annual composition and (d) inter-annual series correction.

\section{B. Night Light Pollution Variation Characteristic at National Sacle}

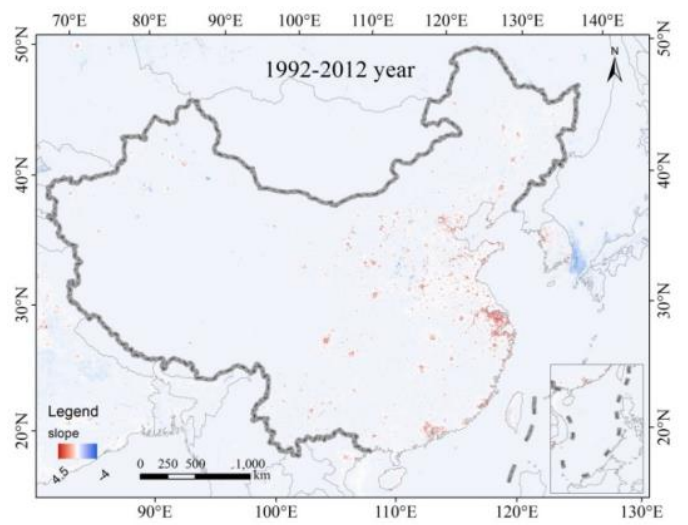

Fig. 3. Change trend of nighttime light pollution in China. (Red color represents light pollution increase, blue color represents light pollution decrease and white color represents light pollution stable).

Linear regression trend method was employed to reveal the change trend of light pollution in China from 1992 to 2012, as shown in Fig. 3. The figure shows that light pollution has experienced sharply increasing trend at national level last year. Increasingly light polluted area mainly locates in municipalities and provincial capitals, whereas a local area shows the decline trend, for example, Shanxi and Xinjiang province. Moreover, most of the area is of stable lighting. It can be divided into two cases: one case locates in the urban core area and another case is the no night light area. The former case is because urban core night light is under saturation from 1992 to 2012, so the night light pollution has significant changes. However, the latter case is almost non-residential area and it lacks night lighting, thus there is almost no night light pollution. China night light pollution mainly locates in three major urban agglomerations (Beijing-Tianjin-Hebei region, Yangtze River Delta and Pearl River Delta), followed by middle Yangtze, Shandong Peninsula Chengdu-Chongqing and Harbin-Changchun. Moreover, the night light pollution in Inner Mongolia, Shanxi, Shaanxi, Henan and Xinjiang also increases significantly. In addition, some urban cities in the north of Shanxi, the south of Inner Mongolia and Xinjiang showed the 
decreasing light pollution significantly.

In order to investigate light pollution of the three major urban agglomerations (Beijing-Tianjin-Hebei region, Yangtze River Delta and Pearl River Delta), the synthesis map (Fig. 4) was generated by using muti-temporal (1992, 2012 and 2012 year) nighttime light imageries. The white color represents stable light pollution from 1992 to 2012, the red color represents extended light pollution and blue color represents decreasing light pollution respectively.

Compared to the Pearl River Delta and Beijing-Tianjin-Hebei region, Yangtze River Delta has experienced rapid and wide light pollution along the Yangtze River and it has formed about $300 \mathrm{~km}$ of light pollution stretch zone from Shanghai to Nanjing. In addition, Hangzhou and Ningbo experienced increasing light pollution Most light polluted cities (Guangzhou, Dongguan, Shenzhen, Hongkong, Macao, Zhuhai and Foshan) show white color in Fig. 4(c), this result indicates that light pollution in Pearl River Delta is more stable from 1992 to 2012. In addition, light pollution in Hongkong decreased, which may relate to Hongkong's light pollution policy in the recent years. Compared with Yangtze River Delta, Beijing, Tianjin and Hebei urban agglomeration nighttime light pollution is mainly located in Beijing and Tianjin stretches area and spatial connectivity of light pollution is less obvious than that in Yangtze River Delta and Pearl River Delta.
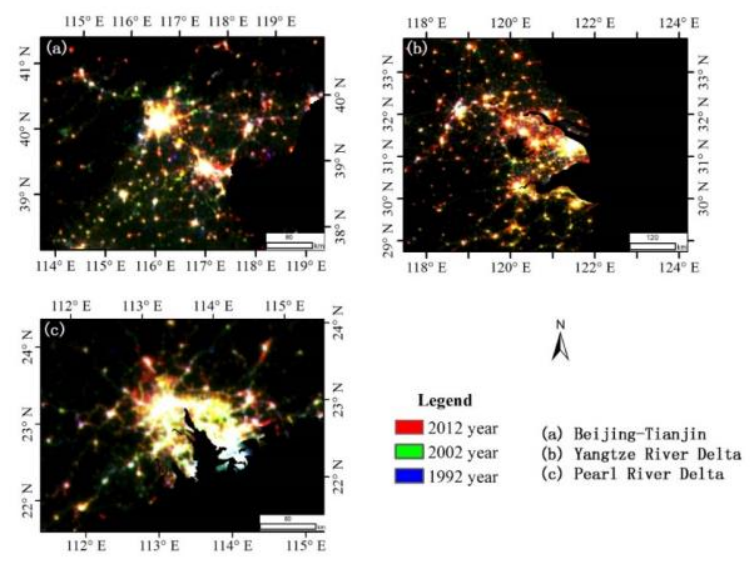

Fig. 4. Color composite of nighttime light of three major urban agglomerations in China. (a) Beijing-Tianjin-Hebei region, (b) Yangtze River Delta and (c) Pearl River Delta.

During the past 21 years, china light pollution has expansion trend (Fig. 3). However, light pollution decline area still exist, which is represented by blue color in Fig. 3. In order to analyze the reasons for the decrease of night light, high resolution satellite images from the Google Earth were selected to validate land cover type with results showed in Fig. 5. Where $\mathrm{A}$ and $\mathrm{B}$ represent oil mining abandoned facilities ; C, D, E and F represent coal mining abandoned facilities; $\mathrm{H}$ and I represent dam construction facilities. It can be found that the main reason of light pollution decrease was abandoned facilities of resource exploitation and the completion of the dam project. In the process of oil exploration, gas flaring phenomenon will happen. Elvidge $e t$ al. [11] has demonstrated that DMSP/OLS can be used to monitor the gas flaring. In the gas flaring area, the gray value of nightlight imagery was close to the urban core gray value and even saturated. When the oil exploration decreases, the gray value of image corresponding to gas flaring area will decrease significantly and the regression change analysis showed a downward trend. The main reason of light pollution decrease in Shanxi, Shaanxi and Inner Mongolia region was that coal exploitation weakened or even exhausted and it resulted in decreasing population and economic activities surrounding the mine, so the nighttime light pollution decreased. Nighttime light pollution decline in Yunnan was mainly due to dam construction. During its construction, the high power searchlights would be used and it would result in strong light pollution. When the project completed, the searchlight cannot be used and the nighttime light intensity decreased, so light pollution change trend decreased.
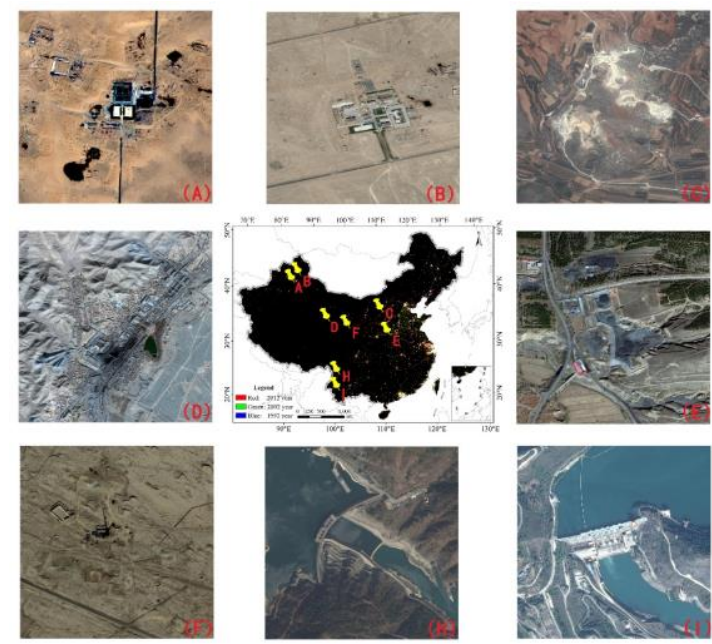

Fig. 5. Validation of the nighttime light pollution decline regions (A and B represent oil mining abandoned facilities ; C, D, E and F represent coal mining abandoned facilities; $\mathrm{H}$ and I represent dam construction facilities).

\section{CONCLUSION}

In order to measure the trend of the light pollution in China, this paper proposed process flow to correct the time series DMSP/OLS nighttime light imagery from 1992 to 2012. Then, linear regression trend method was employed to the temporal and spatial characteristics of light pollution at national scale.

1) After image correction, time series DMSP/OLS nighttime light imagery error can be eliminated efficiently. It improved the consistency of the image and lay foundation for the light pollution analysis.

2) Light pollution increase area mainly locates in municipalities and capital cities and formed Yangtze River Delta, Pearl River Delta and Beijing-Tianjin-Hebei region stretching area. Due to resource depletion and dam construction project, local area light pollution decrease in Shanxi, Inner Mongolia, Shaanxi, Xinjiang and Yunnan provinces.

In this study, DMSP/OLS nighttime light remote sensing was used to demonstrate the change trend of the China light pollution at national scale. It is helpful to objectively and accurately understand the light pollution problems during the past rapid urbanization. Moreover, this conclusion can benefit policy making and light pollution regulation for the government. For the next step, we will further research night light pollution's effect on ecological environment and health risk by integrating multi-source remote sensing data and ground investigation data. 


\section{ACKNOWLEDGMENT}

This research was financially supported by The National Key Research and Development Program of China -Rapid production method of large scale global change products (2016YFA0600302), Hainan Provincial Department of Science and Technology under Grant No.ZDKJ2016021.

\section{REFERENCES}

[1] K. W. Riegel, "Light pollution," Science, vol. 179, no. 4080, pp. 1285-1291, 1973.

[2] J. Bennie, T. W. Davies, J. P. Duffy et al., "Contrasting trends in light pollution across Europe based on satellite observed night time lights," Sci. Rep, vol. 4, Jan 2014.

[3] T. Raap, R. Pinxten, and M. Eens, "Light pollution disrupts sleep in free-living animals," Sci. Rep, vol. 5, Sep 2015.

[4] K. J. Gaston, J. Bennie, T. W. Davies et al., "The ecological impacts of nighttime light pollution: a mechanistic appraisal," Biol.Rev, vol. 88, no. 4, pp. 912-927, Nov 2013.

[5] A. Rodriguez, D. Garcia, B. Rodriguez et al., "Artificial lights and seabirds: is light pollution a threat for the threatened Balearic petrels?" J. Ornithol, vol. 156, no. 4, pp. 893-902, Oct 2015.

[6] P. Cinzano, F. Falchi, C. D. Elvidge et al., "The artificial night sky brightness mapped from DMSP satellite Operational Linescan System measurements," Mon. Not. R. Astron. Soc, vol. 318, no. 3, pp. 641-657, Nov. 2000.

[7] J. Bennie, J. P. Duffy, T. W. Davies et al., "Global trends in exposure to light pollution in natural terrestrial ecosystems," Remote Sens, vol. 7, no. 3, pp. 2715-2730, Mar 2015.

[8] C. Chalkias, M. Petrakis, B. Psiloglou et al., "Modelling of light pollution in suburban areas using remotely sensed imagery and GIS," J.Environ.Manag, vol. 79, no. 1, pp. 57-63, Apr 2006.

[9] C. C. M. Kyba, and F. Hoelker, "Do artificially illuminated skies affect biodiversity in nocturnal landscapes?" Landsc.Ecol, vol. 28, no. 9, pp. 1637-1640, Nov 2013.

[10] Y. Deng, S. H. Liu, and J. M. Cai et al., "Spatial pattern and its evolution of Chinese provincial population and empirical study," Acta Geogr Sinica, vol. 69, no. 10, pp. 1473-1486, 2014.

[11] C. D. Elvidge, D. Ziskin, K. E. Baugh et al., "A fifteen year record of global natural gas flaring derived from satellite data," Energies, vol. 2, no. 3, pp. 595-622, Sep 2009.

[12] M. G. Ma, J. Wang, and X. M. Wang, "Advance in the inter-annual variability of vegetation and its relation to climate based on remote sensing," J. Remote Sens, vol. 10, no. 3, pp. 421-431.2006.

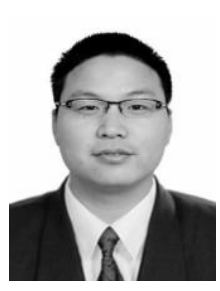

Wei Jiang was born in Hubei, China, in 1991. He received the B.Sc. degree in remote sensing from Fujian Normal University, Fuzhou, in 2013 and the M.Sc.degree in cartography and geographic information systems from the Institute of Remote Sensing and Digital Earth, Chinese Academy of Sciences (CAS), Beijing, China, in 2016, where he has been working toward the $\mathrm{Ph} . \mathrm{D}$. degree in the Institute of
Remote Sensing and Digital Earth since September 2016. His current research interests include nighttime light remote sensing and global land surface water mapping.

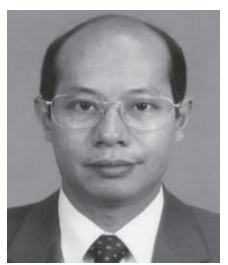

Guojin He was born in Fujian, China, in 1968. He received the B.Sc. degree in geology from Fuzhou University, Fuzhou, China, in 1989, the M.Sc. degree in remote sensing of geology from China University of Geosciences, Wuhan, China, in 1992, and the Ph.D. degree in geology from the Institute of Geology, Chinese Academy of Sciences (CAS), Beijing, China, in 1998. From 1992 to 2007, he was with the Information Processing Department, China Remote Sensing Satellite Ground Station (RSGS), CAS. In 2001, he became the Deputy Director of the Information Processing Department, RSGS, CAS. Since 2004, he has been a Professor and the Director of the Information Processing Department, RSGS, and also the head of the research group of Remote Sensing Information Mining and Intelligent Processing. From 2008 to 2012, he was a Professor and the Director of the Value-added Product Department and the Deputy Director of the Spatial Data Center, Center for Earth Observation and Digital Earth, CAS. Since 2013, he has been a Professor and the Director of the Satellite Data Based Value-added Product Department and the Deputy Director of RSGS, Institute of Remote Sensing and Digital Earth, CAS. A large part of his earlier research dealt with information processing and applications of satellite remote sensing data. His current research interests are focused on optical high-resolution remote sensing image understanding as well as using information retrieved from satellite remote sensing images in combination with other sources of data to support better understanding of the Earth.

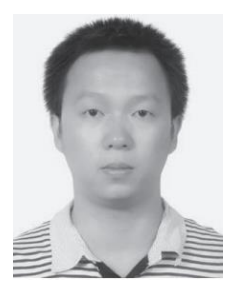

Tengfei Long was born in Wuhan, China, in 1986. He received the B.Sc. degree in remote sensing from Wuhan University, Wuhan, in 2008 and the M.Sc. degree in cartography and geographic information systems from the Center for Earth Observation and Digital Earth, Chinese Academy of Sciences (CAS),Beijing, China, in 2011, where he has been working toward the Ph.D. degree in the Institute of Remote Sensing and Digital Earth since September 2013.In 2011, he joined the Institute of Remote Sensing and Digital Earth, CAS, as a Research Assistant. His current research interests include spatial photogrammetry, image registration, land-cover change detection, and computer vision. 